\title{
СВОЙСТВА БАИЕСОВСКИХ ПРОЦЕДУР ОЦЕНИВАНИЯ СРЕДНЕГО НОРМАЛЬНОГО ЗАКОНА ПРИ ОГРАНИЧЕНИЯХ НА КЛАСС АПРИОРНЫХ РАСПРЕДЕЛЕНИЙ
}

(Представил Н. Алумяэ)

Рассмотрим задачу байесовского оценивания среднего нормального закона при условии, что априорное распределение принадлежит фиксированному классу распределений. Аналогичная задача рассматривалась в [ $\left.{ }^{1}\right]$ для оценивания случайного параметра схемы Бернулли. Сформулируем рассматриваемую задачу. Пусть $x_{1}, x_{2}, \ldots, x_{n}-$ независимые реализации случайной величины $X$, распределенной по нормальному закону $N\left(\theta, \sigma^{2}\right)$, причем $\theta$ - независимая от $X$ случайная величина, не меняющаяся в ряду наблюдений, а $\sigma^{2}-$ известный коэффициент. Относительно распределения $F$ величины $\theta$ известно лишь, чTO

1) $F(t)+F(-t+0)=1, \quad t>0$;

2) $F(a)=1, \quad 0<a<\infty$;

3) $F(t)$ вогнута при $t>0$.

Класс таких распределений для заданного $a$ обозначим через $\mathcal{F}(a)$. Качество оценочной процедуры $\delta\left(x_{1}, x_{2}, \ldots, x_{n}\right)$, используемой при оценивании $\theta$, определим в виде

$$
m(\delta, a)=\sup _{-a \leqslant \theta \leqslant a} E_{\theta}\left(\delta\left(x_{1}, x_{2}, \ldots, x_{n}\right)-\theta\right)^{2} .
$$

Ограничимся рассмотрением оценочных процедур $\delta\left(x_{1}, x_{2}, \ldots, x_{n}\right)$, образующих множество $B(a)$ всех байесовских оценок параметра $\theta$ относительно распределений класса $\mathcal{F}(a)$.

Нами исследованы некоторые свойства процедур из класса $B(a)$, используемые при построении $\mathcal{F}(a)$-минимаксной оценки $\delta_{0}$, т. удовлетворяющей равенству

$$
\inf _{\delta \in B(a)} m(\delta, a)=m\left(\delta_{0} ; a\right) .
$$

Так как $\sum_{i=1}^{n} x_{i}$ достаточная статистика, то можно считать, не умаляя общности, что мы имеем дело с одним наблюдением, распределенным по нормальному закону с плотностью $1 / \sqrt{2 \pi} \exp \left(-\frac{(x-\theta)^{2}}{2}\right)$. Для квадратичного риска байесовская оценка относительно распределения $F$ имеет вид $\left[{ }^{3}\right]$ 


$$
\delta_{F}(x)=\frac{\int_{-\infty}^{\infty} \theta g(x-\theta) d F(\theta)}{\int_{-\infty}^{\infty} g(x-\theta) d F(\theta)},
$$

а в случае если $F \in \mathcal{F}(a)$, это можно записать в виде

$$
\delta_{F}(x)=\frac{\int_{0}^{a} \theta[g(x-\theta)-g(x+\theta)] d F(\theta)}{\int_{0}^{a}[g(x-\theta)+g(x+\theta)] d F(\theta)},
$$

где $g(x-\theta)$ плотность распределения наблюдения.

Следующие далее леммы $1-5$ справедливы для любой строгоодновершинной (с. о. в.) $\left[{ }^{2}\right]$ четной плотности $f(x)$, т. е. такой, что $\ln f(x)-$ четная, вопнутая при $x>0$ функция. Очевидно, что таковой является и нормальная плотность.

Л ем м а 1. Пусть $s>t>0$. Тогда для $x>0$ и с. о. в. $f(\cdot)$

$$
s \frac{f(s-x)-f(s+x)}{f(s-x)+f(s+x)} \geqslant t \frac{f(t-x)-f(t+x)}{f(t-x)+f(t+x)} .
$$

Доказательство. В силу вогнутости $\ln f(x)$ для $s>t>0$ $\frac{f(s+x)}{f(s-x)} \leqslant \frac{f(t+x)}{f(t-x)}$, и следовательно, справедливо неравенство

$$
\begin{gathered}
s \frac{f(x-s)-f(x+s)}{f(x-s)+f(x+s)}=s \frac{1-\frac{f(x+s)}{f(x-s)}}{1+\frac{f(x+s)}{f(x-s)}} \geqslant t \frac{1-\frac{f(t+s)}{f(t-s)}}{1+\frac{f(t+s)}{f(t-s)}}= \\
=t \frac{f(x-t)-f(x+t)}{f(x-t)+f(x+t)},
\end{gathered}
$$

что и требовалось доказать.

Л ем м 2. Если $F \in \mathcal{F}(a)$, то для $x \geqslant 0$ и с. о. в. $f(x)$

$$
\delta_{F}(x) \leqslant a \frac{f(x-a)-f(x+a)}{f(x-a)+f(x+a)} .
$$

Док а з а тельст во. Используя представление (2), получаем

$$
\begin{aligned}
\delta_{F}(x) & =\frac{\int_{0}^{a}[f(x-\theta)+f(x+\theta)] \frac{\theta[f(x-\theta)-f(x+\theta)]}{f(x-\theta)+f(x+\theta)} d F(\theta)}{\int_{0}^{a}[f(x-\theta)+f(x+\theta)] d F(\theta)} \leqslant \\
& \leqslant \max _{0 \leqslant \theta \leqslant a} \theta \frac{f(x-\theta)-f(x+\theta)}{f(x-\theta)+f(x+\theta)}=a \frac{f(x-a)-f(x+a)}{f(x-a)+f(x+a)}
\end{aligned}
$$

где последнее равенство следует из леммы 1.

Л ем м а 3. Пусть $\delta_{1}, \delta_{2} \in B(a)$. Если при всех $x \geqslant 0 \quad \delta_{1}(x) \geqslant \delta_{2}(x)$, то $R\left(\delta_{1}, a\right) \leqslant R\left(\delta_{2}, a\right)$, где $R(\delta, \theta)=E \theta(\delta(x)-\theta)^{2}$.

Док аз ательство, С учетом нечетности $\delta(x)$, очевидной из $(2)$, имеем 


$$
R(\delta, a)=\int_{0}^{\infty}\left[(\delta(x)-a)^{2} f(x-a)+(\delta(x)+a)^{2} f(x+a)\right] d x .
$$

В силу леммы 2 при $x \geqslant 0$

$$
\frac{\delta_{1}(x)+\delta_{2}(x)}{2} \leqslant a \frac{f(x-a)-f(x+a)}{f(x-a)+f(x+a)},
$$

откуда простыми преобразованиями получаем:

$$
f(x-a)\left[\delta_{1}(x)+\delta_{2}(x)-2 a\right] \leqslant-f(x+a)\left[\delta_{1}(x)+\delta_{2}(x)+2 a\right] .
$$

Домножая на $\delta_{1}(x)-\delta_{2}(x) \geqslant 0$, имеем

$$
\begin{aligned}
& {\left[\delta_{1}(x)-\delta_{2}(x)\right]\left[\delta_{1}(x)+\delta_{2}(x)-2 a\right] f(x-a) \leqslant} \\
& \leqslant\left[\delta_{2}(x)-\delta_{1}(x)\right]\left[\delta_{1}(x)+\delta_{2}(x)+2 a\right] f(x+a)
\end{aligned}
$$

или

$$
\begin{aligned}
& f(x-a)\left[\delta_{1}^{2}(x)-2 a \delta_{1}(x)\right]+f(x+a)\left[\delta_{1}^{2}(x)+2 a \delta_{1}(x)\right] \leqslant \\
& \leqslant f(x-a)\left[\delta_{2}^{2}(x)-2 a \delta_{2}(x)\right]+f(x+a)\left[\delta_{2}^{2}(x)+2 a \delta_{2}(x)\right] .
\end{aligned}
$$

Прибавляя к обеим частям $a^{2}[f(x-a)+f(x+a)]$ и интегрируя по $x$, получаем требуемое неравенство.

Пусть $P$ и $Q$ - распределения из $\mathcal{F}(a), \Delta=\{\theta, \eta): 0 \leqslant \theta \leqslant \eta \leqslant a\}$. Будем говорить, что $Q$-распределение равномернее $P$-распределения $(P \prec Q)$, если для любой положительной на $\Delta$ и измеримой функции $\omega(\theta, \eta)$ имеет место неравенство

$$
\int_{\Delta} \int \omega(\theta ; \eta)[d P(\theta) d Q(\eta)-d P(\eta) d Q(\theta)] \geqslant 0 .
$$

3 а меч а н ие. Если распределения $P$ и $Q$ обладают плотностями $p$ и $q$ соответственно, то отношение $P \prec Q$ эквивалентно монотонному убыванию функции $\frac{p(s)}{q(s)}$ при $s>0$.

Лемм а 4. Если $P<Q$, то при $x \geqslant 0$ и с. о. в. $f(x)$

$$
\delta_{P}(x) \leqslant \delta_{Q}(x) .
$$

Док а з ательство. Введем обозначения $U_{x}(s)=s[f(x-s)-$ $-f(x+s)], V_{x}(s)=s[f(x-s)+f(x+s)]$. В соответствии с представлением (2)

$$
\delta_{P}(x)=\frac{\int_{0}^{a} U_{x}(\theta) d P(\theta)}{\int_{0}^{a} V_{x}(\theta) d P(\theta)}, \quad \delta_{Q}(x)=\frac{\int_{0}^{a} U_{x}(\eta) d Q(\eta)}{\int_{0}^{a} V_{x}(\eta) d Q(\eta)} .
$$

Пусть $\omega_{x}(\theta, \eta)=U_{x}(\eta) V_{x}(\theta), \quad M=\{(\theta, \eta): 0 \leqslant \theta, \eta \leqslant a\}, \Delta^{\prime}=M \backslash \Delta$. Тогда имеем

$$
\begin{aligned}
& \int_{M} V_{x}(\theta) V_{x}(\eta) d P(\theta) d Q(\eta)\left[\delta_{P}(x)-\delta_{Q}(x)\right]= \\
= & \int_{M} \int_{x}(\eta, \theta) d P(\theta) d Q(\eta)-\int_{M} \int_{x}(\theta, \eta) d P(\theta) d Q(\eta)= \\
= & \int_{\Delta}\left[\omega_{x}(\eta, \theta)-\omega_{x}(\theta, \eta)\right] d P(\theta) d Q(\eta)+ \\
& +\int_{\Delta^{\prime}}\left[\omega_{x}(\eta, \theta)-\omega_{x}(\theta, \eta)\right] d P(\theta) d Q(\eta) .
\end{aligned}
$$


Интегрирование по $\Delta^{\prime}$ меняем на интегрирование по $\Delta$ заменой переменных $\theta=\eta$ и $\eta=\theta$, после чего правая часть в (4) превращается в

$$
\int_{\Delta}\left[\omega_{x}(\eta, \theta)-\omega_{x}(\theta ; \eta)\right][d P(\theta) d Q(\eta)-d P(\eta) d Q(\theta)] .
$$

Ho

$$
\omega_{x}(\eta, \theta)-\omega_{x}(\theta, \eta)=V_{x}(\theta) V_{x}(\eta)\left[\frac{U_{x}(\eta)}{V_{x}(\eta)}-\frac{U_{x}(\theta)}{V_{x}(\theta)}\right] \geqslant 0,
$$

в силу леммы 1 и того, что $\eta \geqslant \theta \geqslant 0$, так как интегрирование ведется по $\Delta$. Кроме того, по условию $P<Q$, а следовательно, выражение (5) неотрицательно, что и доказывает лемму.

Лемм а 5. Если $P \in \mathcal{F}(a)$, то $P\left\langle P_{a} \in \mathcal{F}^{*}(a)\right.$, где $P_{a}-$ равномерное на $[-a, a]$ распределение.

Доказательство. Очевидно, что достаточно доказать справедливость неравенства (3) в случае, когда $\omega-$ характеристическая функция произвольного прямоугольника $\left[\theta, \theta+h_{1}\right]\left[\eta, \eta+h_{2}\right]$, принадлежащего области $\Delta$. В этом случае получаем

$$
\begin{aligned}
& \quad \iint_{\Delta} \omega(\theta, \eta)[d P(\theta) d Q(\eta)-d P(\eta) d Q(\theta)]= \\
& =h_{2}\left[P\left(\theta+h_{1}\right)-P(\theta)\right]-h_{1}\left[P\left(\eta+h_{2}\right)-P(\eta)\right]= \\
& =h_{1} h_{2}\left[\frac{P\left(\theta+h_{1}\right)-P(\theta)}{h_{1}}-\frac{P\left(\eta+h_{2}\right)-P(\eta)}{h}\right] \geqslant 0 \quad \forall h_{1}, h_{2}>0,
\end{aligned}
$$

так как $P(t)-$ вогнутая функция и $\theta \leqslant \theta+h_{1} \leqslant \eta \leqslant \eta+h_{2}$.

В следующей лемме используется конкретный вид строгоодновершинной плотности.

Л ем м а 6. Пусть $f(x)=1 / \sqrt{2 \pi} e^{-\frac{x^{2}}{2}}$, тогда

$$
\max _{-a \leqslant \theta \leqslant a} R\left(\delta_{P_{a}}, \theta\right)=R\left(\delta_{P_{a}}, a\right),
$$

где $\sigma_{P_{a}}$ - байесовская оценка, соответствующая равномерному на $[-a, a]$ распределению.

$$
\int^{a} \theta f(x-\theta) d \theta
$$

Дока з ательство. Так как $\delta_{P_{a}}(x)=\frac{}{\int_{-a}^{a} f(x-\theta) d \theta}$, запишем

$R\left(\delta_{P_{a}}, s\right)$ в виде

$$
\begin{array}{r}
R\left(\delta_{P_{a}} ; s\right)=\frac{1}{\sqrt{2 \pi}} \int_{-\infty}^{\infty}\left[\frac{f_{1}(x-s) \int_{-a}^{a}(s-\theta) f_{1}(x-\theta) d \theta}{\left(\int_{-a}^{a} f_{1}(x-\theta) d \theta\right)^{2}} \times\right. \\
\left.\times \int_{-a}^{a}(s-\theta) f_{1}(x-\theta)\right] d x
\end{array}
$$

где $f_{1}(x)=\sqrt{2 \pi} f(x)$. Нетрудно убедиться в равенстве 


$$
\frac{d}{d x}\left(\frac{f_{1}(x-s)}{\int_{-a}^{a} f_{1}(x-\theta) d \theta}\right)=f_{1}(x-s) \frac{\int_{-a}^{a}(s-\theta) f_{1}(x-\theta) d \theta}{\left(\int_{-a}^{a} f_{1}(x-\theta) d \theta\right)^{2}},
$$

используя которое, получаем из (6) интегрированием по частям следующее равенство

$$
R\left(\delta_{P_{a}}, s\right)=\frac{1}{\sqrt{2 \pi}} \int_{-\infty}^{\infty}\left[\frac{f_{1}(x-s)}{\int_{-a}^{a} f_{1}(x-\theta) d \theta}\left(\int_{-a}^{a} f_{1}(x-\theta)(s-\theta)(x-\theta) d \theta\right)\right] d x
$$

Вновь интегрируя по частям, имеем

$$
\begin{gathered}
R\left(\delta_{P_{a}}, s\right)=\frac{1}{\sqrt{2 \pi}} \int_{-\infty}^{\infty} \frac{f_{1}(x-s)}{\int_{-a}^{a} f_{1}(x-\theta) d \theta}\left[\left.(s-\theta) f_{1}(x-\theta)\right|_{-a} ^{a}+\int_{-a}^{a} f_{1}(x-\theta)\right] d x= \\
=1+\frac{1}{\sqrt{2 \pi}} \int_{-\infty}^{\infty} \frac{(s-a) f_{1}(x-a)-(s+a) f_{1}(x+a)}{\int_{-a}^{a} f_{1}(x-\theta) d \theta} f_{1}(x-s) d x
\end{gathered}
$$

откуда путем простых преобразований получаем

$$
\begin{gathered}
R\left(\delta_{P_{a}}, s\right)=1-\frac{1}{\sqrt{2 \pi}} f_{1}(a) f_{1}(s) \times \\
\times \int_{0}^{\infty} \frac{(a-s) \operatorname{ch} \frac{a+s}{2} x+(a+s) \operatorname{ch} \frac{a-s}{2} x}{\int_{0}^{a} f_{1}(\theta) \operatorname{ch} \frac{\theta x}{2} d \theta} f_{1}(x) d x .
\end{gathered}
$$

Но $f(a) \leqslant f(s)$ для $s \leqslant a$ и сh $t \geqslant 1 \forall t$.

Следовательно,

$$
R\left(\delta_{P_{a}}, s\right) \leqslant 1-\frac{1}{\sqrt{2 \pi}} 2 a f_{1}^{2}(a) \int_{0}^{a} \frac{f(x) d x}{\int_{0}^{a} f(\theta) \operatorname{ch} \frac{\theta x}{2} d \theta}
$$

а последнее выражение есть $R\left(\delta_{P_{a}}, a\right)$, что и доказывает лемму 6 .

Т е о р ем а. Оценка $\delta_{P_{a}}$ является $\mathcal{F}(a)$-минимаксной, т. е. удовлетворяющей равенству (1).

Дока зательство. В силу лемм 4 и 5 при $x \geqslant 0 \quad \delta_{F}(x) \leqslant \delta_{P_{a}}(x)$, откуда по лемме 3

$$
\max _{-a \leqslant \theta \leqslant a} R\left(\delta_{F}, \theta\right) \geqslant R\left(\delta_{F}, a\right) \geqslant R\left(\delta_{P_{a}}, a\right),
$$

а использование леммы 6 завершает доказательство теоремы. 


\section{Л И Т Е Р А Т Р А}

1. Ольман В. Изв. АН ЭССР. Физ. Матем., 33, № 3, 285-290 (1984).

2. Феллер В. Введение в теорию вероятностей и ее приложения. М., «Мир», 1967.

3. Вальд А. Статистические решающие функции. - В кн.: Позиционные игры. М., «Наука», 1967.

Ннститут кибернетики

Академии наук Эстонской ССР
Поступила в редакцию $1 /$ XII 1983

\section{A. SMUNDAK, V. OLMAN}

\section{NORMAALJAOTUSE KESKVÄARTUSE BAYESI TUUPI HINNANGUD KITSENDUSTEGA APRIOORJAOTUSTE KLASSI KORRAL}

On vaadeldud normaaljaotuse keskväärtuse Bayesi tüüpi hinnanguid. Aprioorjaotuste klass koosneb fikseeritud lōplikul lõigul $[-a, a]$ määratud sümmeetrilistest ja lõigul $[-a, 0]$ kumeratest jaotustest. On otsitud hinnangut, mis minimiseeriks ruutkeskmise riski maksimumi lōigul $[-a, a]$, ja tōestatud, et selleks on Bayesi hinnang $\delta_{a}$, mis vastab ühtlasele aprioorjaotusele. On näidatud, et hinnang $\delta_{a}$ majoreerib poolsirgel $[0, \infty]$ kõiki teisi vaadeldava klassi hinnanguid ja $\delta_{a}$ korral on ruutkeskmise riski väärtus punktides $-a$ ja $a$ minimaalne.

\section{A. SHMUNDAK, V. OLMAN}

\section{PROPERTIES OF BAYES ESTIMATORS OF THE MEAN NORMAL DISTRIBUTION UNDER RESTRICTIONS ON THE CLASS OF A PRIORI DISTRIBUTIONS}

The problem of Bayes estimation of the mean value of normal distribution is considered. It is assumed that the a priori distribution $F(\theta) \in \mathcal{F}(a)$, i. e. $F(\theta)$ is 1) symmetrical, 2) concentrated on the interval $[-a, a]$ for fixed $a>0,3)$ convex on the $[-a, 0]$. The problem is to find the Bayes estimator $\delta$ which corresponds to the a priori distribution from the is to find the Bayes estimator $\delta$ which corre

described class and minimizes the $a \leqslant \theta \leqslant a E_{\theta}(\delta(x)-\theta)^{2}, x \sim N(\theta, 1)$. It is shown that the $\delta_{a}$ corresponding to the uniform distribution on the $[-a, a]$, is the optimal decision. In addition, it is shown that $\delta_{a}$ dominates any other Bayes estimator from this class on the interval $[0, \infty]$, and that the mean square risk at points $-a$ and $a$ is the smallest in case of $\delta_{a}$. 